\title{
Fundamentos y avances del Centro Público de Mediación \#156, con sede en el Centro Universitario de Ciencias Económico Administrativas, Universidad de Guadalajara
}

\author{
The creationand advances of Public Mediation Center \\ \#156 at the University Center for Economic and \\ Administrative Sciences, University of Guadalajara
}

\author{
Agustín Mora López ${ }^{1}$ \\ Universidad de Guadalajara \\ agustin.mora5102@alumnos.udg.mx \\ Sandra Paredes Baltazar ${ }^{2}$ \\ Universidad de Guadalajara \\ sandra.paredes@cucea.udg.mx
}

Fecha de recepción: 17/10/2021

Fecha de aceptación: 23/11/2021

\section{Resumen}

Este trabajo presenta una síntesis sobre los orígenes, propósitos y primeros logros de un centro público de mediación que se formó y opera dentro del ámbito universitario. Promovido desde el Departamento de Ciencias Sociales y Jurídicas de la Universidad de Guadalajara y acreditado por Instituto de Justicia Alternativa del estado de Jalisco, el Centro Público de Mediación número 156, alcanzó tres años de operaciones dentro del Centro Universitario de Ciencias Económico Administrativas. En ese contexto, la presente intervención busca poner de relieve las necesidades que motivaron la creación del organismo, el proceso que siguió su constitución y las bases bajo las que opera. Además, se presenta un apartado que expone algunas de las experiencias que ha reunido el Centro durante este tiempo, sus modos de trabajar, algunos de sus logros y las asignaturas pendientes que se tienen hasta la fecha. El objetivo final es compartir con los lectores este tipo de iniciativas públicas, las cuales aspiran a difundir y llevar a la práctica los 
Métodos Alternos de Solución de Conflictos, así como contribuir a la construcción de una cultura de paz.

Palabras clave: Centro Público de Mediación, Métodos Alternos de Solución de Conflictos, CUCEA.

\section{Abstract}

This article presents a synthesis of the origins, objectives, and early achievements of a public center for mediation that was created and functioned on a university campus. Promoted by the Department of Social and Juridical Sciences of the University of Guadalajara and accredited by the Institute of Alternative Justice of the state of Jalisco, Centro Público de Mediación no. 156 has operated at the University's Center for Economic and Administrative Sciences for three years. The analysis of that setting elucidates the needs that led to the creation of the Centro, the process that ensued upon its constitution, and the bases under which it functioned, including a section that expounds some key experiences in which the Centro has participated during this time, its methods of operation, some of its achievements, and specific issues that are pending at present. The objective is to inform readers about this kind of public initiative, which exists to disseminate Alternative Methods of Conflict Resolution, put them into practice, and in this way contribute to the construction of a culture of peace.

Keywords: Public Center for Mediation, Alternative Methods of Conflict Resolution, CUCEA.

\section{La iniciativa de un Centro Público de Mediación en el entorno universitario}

Los retos actuales de un mundo cada vez más competitivo, obligan a las instituciones de educación superior a infundir entre su comunidad, modelos que apliquen nuevos paradigmas para la solución pacífica de los conflictos que ocurren cotidianamente en su entorno. Los Métodos Alternos de Solución de Conflictos (MASC), fueron impulsados después de eventos tan funestos como las guerras mundiales, para asentar que es posible construir una sociedad que dialoga para comprender y conciliar sus diferencias, antes que recurrir a medidas violentas para imponer la opinión propia sobre otra distinta. A partir de esta premisa, distintos organismos internacionales han contribuido a la creación de alternativas 
resolutivas, al resguardo de los derechos humanos y a impulsar la implementación de acciones positivas que lleven a la materialización de una cultura pacífica.

El avance incesante de estos ideales a nivel global, posibilitó que México realizara la reforma constitucional de 10 de junio de 2011, la cual significó un verdadero cambio en el sistema jurídico y normativo del país. Con ello, las leyes nacionales comenzaron a empatarse con directrices internacionales en torno a la defensa de los derechos humanos. El artículo 17 de la Constitución Política de los Estados Unidos Mexicanos, otorga a los MASC el carácter de derecho humano; convirtiendo en una obligación de los gobiernos brindar opciones no adversariales a los ciudadanos para la resolución de sus controversias. Una ventaja adicional del nuevo sistema de impartición de justicia, es que promueve la cultura de la paz, por lo que resulta de suma importancia considerar el potencial de este modelo, donde la voluntariedad de los involucrados y el acompañamiento de un mediador o mediadora especializados en MASC, puedan llegar a solucionar los conflictos de manera expedita y, sobre todo, protegiendo la parte emocional de las personas que se encuentren en desacuerdo.

En este contexto, parece innegable la relevancia que las universidades y Centros educativos tienen respecto a la labor formativa, de difusión y aplicación de la justicia alternativa; en vista de ser esta una gran oportunidad para armonizar las relaciones humanas y reconstruir el tejido social desde sus integrantes más jóvenes. La Universidad de Guadalajara (UDG) y el Centro Universitario de Ciencias Económico Administrativas (CUCEA), a través del Departamento de Ciencias Sociales y Jurídicas (DCSJ), se comprometieron a cooperar en la formación y aplicación de los métodos pacíficos para la solución de conflictos y se sumaron a la construción de una cultura de paz, de manera formal desde el año de 2017.

En vinculación con el Instituto de Justicia Alternativa del Estado de Jalisco (IJA), el DCSJ comenzó a plantear un programa de largo aliento en materia educativa, de investigación y vinculación con los MASC. Luego de realizar varios diplomados en esta materia para la comunidad universitaria y el público general, se inició el proyecto para conformar un Centro Público de Mediación que operara dentro del Centro Universitario y en adhesión al Departamento de Ciencias Sociales y Jurídicas. Concluidas las solicitudes correspondientes, el 29 de noviembre de 2017, el IJA llevó a cabo la visita requerida para la acreditación del Centro; tras la cual, la Dirección de Acreditación, Certificación y Evaluación del IJA, resolvió otorgar, el 7 de diciembre de 2017, la acreditación oficial a la iniciativa del DCSJ y con ello logró constituirse, finalmente, el Centro Público de Prestación de Servicios de Métodos Alternativos número 156, certificado por el IJA.

La inauguración formal del Centro se dio el 12 de noviembre del 2018. A partir de entonces, ofrece sus servicios tanto a la comunidad del CUCEA, como al público general de zonas aledañas. Tras sus primeros dos años de funcionamiento ininterrumpido, en octubre de 2020, el IJA le otorgó una reacreditación para que siga operando bajo su aval, con una vigencia activa hasta noviembre 
de 2022. De esta manera, el Centro número 156, se convirtió en el primero de su tipo que opera dentro de la Red de Centros Universitarios de la Universidad de Guadalajara. Lo anterior no sólo posiciona al CUCEA a la vanguardia en la oferta de servicios de MASC, sino que también contribuye a resolver, de manera eficiente, económica y ágil, los conflictos que surgen dentro de la comunidad en que están insertos la Universidad y todos sus miembros.

De esta manera, el Centro funciona con el apoyo y participación del CUCEA, en general, y el Departamento de Ciencias Sociales y Jurídicas, en específico, además de estar en vinculación directa con el Instituto de Justicia Alternativa del Estado de Jalisco. Además, tiene la posibilidad de integrar a sus actividades a otros Centros Universitarios de la red UDG y, a través de la celebración de convenios específicos, puede colaborar con otras instituciones públicas y privadas nacionales o extranjeras; siempre en busca de difundir y promover la aplicación de los MASC y contribuir a edificar una cultura de paz.

A nivel educativo, para la UDG resulta pertinente que se brinden este tipo de servicios, ya que se fomenta la adopción de los MASC — mediación, conciliación, arbitraje y negociación- entre sus integrantes y se promueve su enseñanza práctica entre los alumnos prestadores de servicio social, especialmente los que provienen de las licenciaturas de derecho, psicología y trabajo social, quienes suelen auxilian en dicho centro público. De la misma forma, existe la oportunidad para que los egresados del CUCEA formen parte de los arbitrios expertos en ciertas materias de las áreas económico administrativas, al colaborar en el Centro como prestadores de servicio o practicantes, de acuerdo con los requerimientos que indica la Ley de Justicia Alternativa para el Estado de Jalisco.

Desde una perspectiva social, es innegable la necesidad e importancia que tiene contar con espacios de mediación para todos aquellos que necesiten ser orientados por mediadores expertos y certificados en el manejo de conflictos y conciliaciones, además de tratarse de personal ético que preste sus servicios sin fines de lucro. Si tomamos en cuenta que la mediación es un Método Alterno a lo que llamamos justicia tradicional, debe resaltarse que esta acción nos ayuda a resolver controversias entre particulares de una manera menos desgastante, acortando los gastos y el tiempo que implicara un proceso ordinario; aunado a que su aplicación promueve la cultura de paz entre las partes en disputa, al promover el diálogo y la reflexión pacífica para la resolución de sus controversias.

De tal manera, el Centro Público de Prestación de Servicios de Métodos Alternativos número 156, coadyuva en la construcción de la cultura de paz, mediante el tratado de los problemas en un ambiente de conciliación y confianza, llamando a la buena voluntad y a la disposición de las personas, exhortándolas a manejar los problemas de una forma diferente, sin enfrentamientos ni violencia y procurando siempre llegar a una solución amistosa.

El Centro de mediación que opera en el CUCEA, trabaja también en diversas actividades de vinculación que favorecen y respaldan su trabajo, contribuyendo además a la extensión de los principios que persigue. Entre otras acciones, pueden mencionarse las iniciativas siguientes: 
- Colaboración con el Programa de Cultura de Paz del CUCEA.

- Manejo de redes sociales (Facebook) propias para la interacción y guía de usuarios y potenciales solicitantes de los servicios de mediación.

- Asesoría vía telefónica.

- Colaboración con la Red de Apoyo Psicológico del municipio de Zapopan.

- Colaboración con el Consejo Ciudadano de Seguridad de Jalisco.

- Colaboración con el Centro de Justicia para las Mujeres de Jalisco, especialmente para canalizar los casos de violencia.

- Exposiciones y entrevistas en medios de comunicación de la UDG, donde se aborda la existencia y conveniencia de la mediación y la justicia alternativa, además de brindar asesorías certificadas a las preguntas y dudas del público ante problemáticas concretas.

- Organización de múltiples Webinars, con temas relativos a la cultura de paz y la mediación.

- Implementación de asesorías y mediaciones virtuales, como alternativa para la prestación de servicios durante la pandemia provocada por el COVID-19.

\section{Alcances del Centro Público de Prestación de Servicios de Métodos Alternativos}

A diferencia de otros Centros, el número 156, se caracteriza por dar acompañamiento cercano a las partes que inician un proceso mediación. Resulta primordial el considerar cada caso de manera específica y otorgando una atención personalizada; en busca de que los solicitantes se sientan escuchados y acompañados en todo momento, que tengan la seguridad de ser canalizados a las instancias pertinentes en cada situación y puedan sobrellevar el conflicto hasta su resolución. De acuerdo con su organización particular, este Centro funciona a través de una Dirección general y mediante cinco ejes que agrupan sus funciones como organismo. Dichas áreas se articulan de la siguiente manera:

I. Métodos Alternativos de Solución de Controversias.

II. Mediación y Conciliación.

III. Psicología.

IV. Trabajo social.

V. Difusión, vinculación y capacitación.

El área de los Métodos Alternativos de Solución de Controversias, tiene entre sus actividades los siguientes puntos: 
- Acciones de difusión y asesoría para dar a conocer las características y beneficios de los MASC entre las personas que acuden, o pueden acudir, al Centro para atender algún conflicto.

- Poner a disposición de los solicitantes los servicios de asesoría psicológica, con el fin de llevar a cabo las mediaciones de manera sistémica.

- Apoyarse en el área de Trabajo Social para la gestión del proceso de mediación.

- Vincularse con el Programa Integral de Cultura de Paz del CUCEA, realizando un trabajo multidisciplinario que fortalezca la atención a los usuarios universitarios que soliciten apoyo.

El área de Mediación y Conciliación tiene la misión fundamental de brindar asesoría sobre las características de la mediación y la concilación, además de ejecutar y dar acompañamiento a los procesos de esta naturaleza que se efectúen. Normalmente, en el área de viabilidad, se deternmina si el asunto es mediable o definitivamente no. Una vez que se decide comenzar la intervención, el facilitador del Centro, en acuerdo con las partes involucradas, determina el método a seguir para la resolución del conflicto particular.

Según las necesidades de cada caso, se puede comenzar por sugerir una mediación o una conciliación y proceder a su realización; si la situación es demasiado controvertida, ambas partes pueden acceder a la intervención de un árbitro certificado que formule un convenio, al cual deberán apegarse los involucrados. Al final, en los convenios finales aparecen especificados los métodos empleados durante la intervención.

El área psicológica otorga apoyos en esta materia a los involucrados durante los procesos de mediación y resolución de convenios finales; con el objetivo de fortalecer la parte emocional que puede afectarse ante los problemas abordados en cada caso. Así mismo, en esta área se llevan a cabo las escuchas de menores — cuando son requeridas-y las juntas de avenimiento en casos de divorcios administrativos.

Por su parte, el área de trabajo social se enfoca en brindar asesoría e información sobre los diferentes programas gubernamentales y universitarios que hay respecto a la mediación y la cultura de paz. También se encarga de realizar los estudios socioeconómicos de los solicitantes de servicios del Centro, además de organizar conferencias abiertas con temáticas que contribuyen a reforzar la convivencia pacífica entre la comunidad del CUCEA y en pos de la armonía universitaria.

Finalmente la sección de difusión, vinculación y capacitación, se encarga de fomentar la cultura de paz a través de diversos medios. De igual forma, y en colaboración con el Instituto de Justicia Alternativa de Jalisco, buscar difundir los MASC mediante trípticos, programas de radio, charlas y demás estrategias que permiten acercar estos mecanismos de paz con la comunidad universitaria y los usuarios externos del Centro. 
En suma, el Centro Público de Prestación de Servicios de Métodos Alternativos del CUCEA, tiene entre sus propósitos:

- Promover que el profesorado del CUCEA, y otros Centros Universitarios de la UDG, se capaciten en cuanto a los Métodos Alternativos para la Solución de Controversias; se certifiquen por en esta materia por el IJA y contribuyan al desarrollo de las actividades propias del Centro de mediación en cuestión.

- Realizar cursos que fomenten la cultura de paz entre la comunidad universitaria.

- Promover la impartición de diplomados en MASC para que profesionistas de diferentes áreas se capaciten y certifiquen como prestadores de estos servicios.

- Difundir los servicios y beneficios que ofrecen el Centro de mediación y la justicia alternativa, a través de medios tradicionales — radio, televisión, etc. - y redes sociales.

- Ofrecer asesorías en espacios públicos - municipales, universitarios, etc.- sobre la mediación, además de canalizar los casos que puedan ser atendidos en el Centro número 156.

Dada la necesidad de contar con insumos básicos para la operación de este organismo, se han establecido cuotas de recuperación para los servicios prestados, las cuales son muy accesibles para los usuarios. Por una asesoría, se solicitan 50 pesos mexicanos -2.5 dólares, aprox.- mientras que para un proceso de mediación la cuota establecida es de 200 pesos - 10 dólares, aprox.- mensuales hasta la conclusión del caso. La aplicación de estos montos está sujeta a estudios socioeconómicos, por lo cual pueden otorgarse aún descuentos o exenciones en casos particulares.

\section{Algunas experienciastras los primeros avances del Centro}

Debido a las satisfacciones directas que los autores hemos tenido al colaborar dentro del Centro Público de Mediación del CUCEA, queremos aprovechar esta sección para hacer un recuento, en primera persona, sobre los avances y expectativas que ha tenido el organismo a través de sus más de tres años de funcionamiento. En ese sentido, presentamos ahora una especie de síntesis—a manera de preguntas y respuestas-sobre el desarrollo del Centro, visto desde la experiencia particular de la directora de esta unidad mediadora.

¿Cuándo se creó el Centro de Mediación que está en CUCEA y cómo fue el proceso para instituirlo? Este Centro de mediación es el primero en un Centro 
Universitario en la red de la Universidad de Guadalajara. El proceso de creación se llevó a cabo entre 2017 y 2018. Para constituirlo fue necesario hacer una petición al Instituto de Justicia Alternativa del Estado de Jalisco, en la cual nosotros pretendíamos acreditarnos de acuerdo a los procedimientos que marca el Instituto. Para lograr todos los requerimientos, se contó con el apoyo del Departamento de Ciencias Sociales y Jurídicas de la UDG, en el CUCEA, y gracias al trabajo y apoyo de la Jefa del dicho departamento, la Doctora Noemí Silva Gutiérrez, quien acogió este gran proyecto.

En el trayecto, nos dimos a la tarea de buscar un lugar idóneo para lograr la acreditación. Cuando comenzamos el trámite, en noviembre de 2017, nos hacía falta construir toda la infraestructura que nos permitiera cumplir con los requerimientos hechos por el IJA; un sitio con espacios suficientes e incluyentes que permitieran el acceso universal, además de salas acondicionadas y otros equipamientos generales. Todos esos requisitos pudieron cubrirse con el apoyo del mismo DCSJ.

Así, para noviembre de 2018, fue inaugurado de manera formal el Centro Público de Mediación número de 156, acreditado por el IJA. En la ceremonia estuvieron autoridades del Instituto y de todas las instancias del CUCEA que apoyaron este proyecto. Cabe decir que la certificación ante el IJA debe renovarse cada dos años, actualmente tenemos vigente una nueva certificación hasta 2022 y esperamos que, después de esa fecha, podamos renovarla otra vez.

$¿$ ¿Cuáles han sido los mayores retos para el desarrollo y consolidación del Centro de Mediación? Probablemente el mayor reto es el seguir fomentando una cultura de paz en toda la comunidad CUCEA, en primera instancia, así como lograr que toda la población universitaria conozca la existencia de este Centro, los beneficios de los MASC y la utilidad que esto puede tener en su vida, tanto profesional como personalmente. En segundo lugar, está el reto de que haya más mediadores certificados. Para ello, hemos realizado varios diplomados, a los cuales se invita a profesores interesados para que tomen el curso y hagan su procedimiento de certificación. De tal manera, nos parece muy relevante hacer mayor difusión sobre la existencia y utilidad de los MASC, así como propagar la formación de mediadores profesionales.

¿Cuáles son las áreas de oportunidad del Centro? Una de las principales está relacionada con otras iniciativas del DCSJ, especialmente con la maestría que éste ofrece sobre Métodos Alternos de Solución de Conflictos. Esto da la pauta para que los alumnos que la están cursando puedan hacer prácticas aquí y eso nos parece muy relevante. Aunque la teoría es fundamental, poder llevarla a la práctica resulta trascendental y a ello contribuyen ambas instancias; tanto a través de los cursos como del Centro, los estudiantes pueden desarrollar habilidades teórico-prácticas al conocer de cerca los casos que son atendidos ahí por parte de mediadores certificados. Otras áreas de oportunidad están en poder incidir dentro de contextos más amplios, especialmente en pos de la cultura de 
paz. Para ello, es importante aprovechar la difusión que nos permita darle mayor visibilidad a los MASC, además de multiplicar la empatía y la solidaridad dentro de la comunidad específica en que el Centro opera.

¿Cuál es el promedio de casos atendidos por el Centro? Si hacemos una recuento desde noviembre del 2018 a agosto de 2021, encontramos que se han dado aproximadamente 352 asesorías. Las personas atendidas pertenecen tanto a la comunidad CUCEA, como a zonas aledañas y de otros contextos. Cabe mencionar que además de estas, se han brindado otras en el Centro Cultural Constitución, que es un lugar que se acondicionó y depende del Ayuntamiento de Zapopan, ya que existe un convenio por el cual se ofrecen asesorías gratuitas para difundir el Centro de Mediación de CUCEA. De tal manera, la suma total de las asesorías dadas es de 364. Aunque queda mucho por hacer, puede decirse que se está avanzando con los objetivos de dar a conocer los nuevos métodos y el de brindar el apoyo a quienes se encuentran enfrentando alguna situación conflictiva.

En cuanto a los tipos de servicios prestados, el $85 \%$ de estos son asuntos familiares. Debido a la pandemia se ha incrementado considerablemente esta estadística y pueden observarse principalmente asuntos como: conflictos sucesorios, custodias y pensiones alimenticias. También se presentan casos específicos donde existen situaciones más concretas, como cuando las parejas no están casadas y quieren terminar bien su relación o algunas situaciones de violencia en el noviazgo.

¿Para quiénes son estos servicios, cuál es el público objetivo? Al ser un Centro público, los servicios ofrecidos son para toda la sociedad en general. Sin embargo, dado el contexto universitario en que el Centro está inserto, el principal público objetivo es la propia comunidad CUCEA, es decir, el alumno, los profesores y trabajadores de este Centro Universitario, además de los pertenecientes a cualquier otro órgano de la red universitaria. En segundo lugar, se pretende llevar los servicios de mediación a los habitantes de las zonas aledañas de nuestra sede y, en general, de cualquier parte del estado. Sabemos que en la vía jurisdiccional hay competencias y aquí, afortunadamente, este Centro Público atiende a todos los municipios.

¿Cuáles son los tipos de conflictos que atienden mayormente, además de los asuntos familiares? Lamentablemente, el $85 \%$ son de materia familiar y resulta preocupante ver que estos problemas se han complicado, generalmente, por falta de comunicación entre las partes y esto desata una cascada de muchos problemas más. Últimamente, se ha observado otro fenómeno conflictivo dentro del ámbito familiar, a causa de los fallecimientos por COVID-19. Debido a estas ausencias, los roles domésticos cambian repentinamente junto con toda la dinámica familiar previa; dentro CUCEA, por ejemplo, se ha observado un incremento en el ausentismo y deserción por parte de los estudiantes de licenciatura, ya que, ante la falta de alguno de los padres, deben incorporarse al mundo 
laboral de manera emergente. Tristemente, la pandemia ha contribuido a incrementar los asuntos en materia familiar, mayormente como un efecto del súbito cambio de roles que han generado los fallecimientos.

Además de los conflictos de índole familiar, los asuntos relacionados con los adultos mayores representan un número importante de las asesorías brindadas. Se observan situaciones de abandono por parte de los familiares directos, así como una devaluación de valores respecto a su trato general. Lo anterior, nos llama a reforzar al área de trabajo social y a fortalecer las redes de apoyo que podamos brindar, buscando cómo auxiliar a los adultos mayores, adónde se pueden canalizar, en dónde conseguir asistencia médica, psicológica y toda la protección integral que demandan.

¿Porqué considera que una persona, corporación o institución debería tomar en cuenta los MASC y no una vía jurisdiccional tradicional? En primer lugar, es importante considerar que existen diferentes maneras de solucionar los conflictos y que éstos son inherentes a la convivencia de dos personas o más. Por ello, hay que hacer del conocimiento general que existen maneras alternas de solucionar las diferencias, medios independientes de la vía jurídica tradicional. Hay que resaltar, además, que estas rutas alternas permiten evadir los costos y tiempos que demanda un procedimiento convencional, donde la saturación de los juzgados y el costo de un procedimiento pueden alargar demasiado cualquier proceso.

En la esfera personal, los años de un litigio pueden resultar en sentimientos de preocupación, incertidumbre, agonía, o violencia, lo cual compromete en demasía el bienestar de las personas que enfrentan algún conflicto. De tal manera, una opción viable de acortar el sufrimiento y todas las implicaciones personales que puede llevar un conflicto es, precisamente, la adopción de los MASC; cuyo proceso de resolución resulta más expedito y menos oneroso, además de estar acompañado del apoyo personal e integral que brindan los centros de mediación.

Respecto a la adopción de estos métodos por parte de las instituciones, vale poner como ejemplo a la propia Universidad de Guadalajara. Además de su esfuerzo por mantener en funcionamiento al propio Centro número 156, la Casa de Estudios creó un área específica para garantizar los derechos de su comunidad, a través de la Defensoría de los Derechos Universitarios. En CUCEA, igualmente, se cuenta con el Programa integral de Cultura de Paz, cuyo interés es difundir y promover esta postura de desarrollo social. En el Centro Público de Mediación de este Centro Universitario, colaboramos activamente con ambas iniciativas.

Sin duda, la UDG se encuentra preocupada por incidir en que su comunidad logre resolver los conflictos que puedan darse al interior de la institución. Naturalmente, queda aún mucho por hacer en esta materia, pero los avances logrados tanto en la Defensoría como en el Programa de Cultura de Paz del CUCEA, más los propios aportes del Centro de Mediación 156, comienzan a mostrar las ventajas que puede tener para la institución el adoptar los MASC dentro su organi- 
zación. La misión futura, deberá ser lograr que estas instancias y procedimientos sean arropados por toda la comunidad y que llegue a contarse con Centros de mediación, o similares, en cada unidad de la red universitaria.

¿En qué consiste un proceso de mediación? La mediación es un método alterno de resolución de conflicto, en donde un tercero imparcial o un facilitador de la comunicación acompaña a las personas para que ellos mismos formen un acuerdo que termine el enfrentamiento. Los terceros que fungen como mediadores o facilitadores, deben certificarse como tal y operar al margen de la normatividad vigente. En el caso de Jalisco, el IJA, permite que los egresados de cualquier licenciatura se formen como mediaciones, a diferencia de otros estados donde la prerrogativa está reservaba para los abogados.

En un proceso de medición, debe tenerse claro que la función de los intermediarios es facilitar la comunicación entre las partes enfrentadas, no proponer o imponer las resoluciones finales. Por su parte, la conciliación es un método alterno donde igualmente se facilita la comunicación, pero en el cual el intermediario sí opina y sugiere alguna resolución que beneficie ambas partes. Comúnmente, son los abogados quienes fungen como conciliadores, mientras que en las mediaciones pueden intervenir diversos perfiles profesionales, como pueden ser docentes, médicos, psicólogos o trabajadores sociales.

¿Cuál es el perfil que debe tener un mediador y cuáles deberían ser sus competencias y habilidades? Lo primero que debe tener un mediador es empatía, saber escuchar a las personas y comprender lo que están sintiendo sin juzgar. Es muy importante tener esa sensibilidad hacia lo que siente el otro que está viviendo el conflicto. Lo anterior es un elemento importante para poder parafrasear, es decir, para devolverle al otro lo que se entendió.

En ese perfil de mediador, es necesario tratar ser imparcial a toda costa, lo cual puede resultar algo muy complejo, dada la naturaleza humana que se apoya en la empatía y paciencia hacia su interlocutor. Sin duda, dicha habilidad es algo muy importante en un mediador familiar, es recomendable que se tenga desarrollada alguna competencia de inteligencia emocional, para manejar sus emociones y poder comprender a los mediados cabalmente.

Entre las habilidades mínimas que debe reunir cualquier facilitador, están: la escucha activa empática, el parafraseo y la paciencia. La mediación es una combinación de arte y ciencia, ya que hay una base científica en todo lo que está estudiado y probado; es un arte, igualmente, porque implica una destreza propia del mediador. Ambas bases técnicas deben estar claras y bien estructuradas para el mediador. También, dentro de las reglas de la mediación, está el respeto y corresponde al mediador monitorear que éste se cumpla en todo momento.

¿Cómo funciona el parafraseo y la escucha activa? La escucha activa empática es estar consciente de lo que pasa durante la sesión de mediación. Se trata de que el facilitador esté comprometido cien por ciento con la mediación, que esté escuchando de manera consciente y no únicamente oyendo. Para ello, se recomienda estar convencidos de la utilidad de los métodos alternos, desarrollar 
la sesión en un lugar específico y con circunstancias determinadas, tales como contar con una mesa redonda, estar entre paredes pintadas en tonos tenues y todo un contexto de tranquilidad que permita el buen entendimiento de todas las partes.

Entonces, al hacer una escucha activa estamos siendo capaces del parafraseo. En esta técnica, el mediador debe verbalizar aquello que se ha escuchado por parte de alguno de los mediados, pero dicho de tal manera que exprese fielmente lo que aquel externó durante su intervención. El parafraseo tiene dos elementos muy importantes, por un lado, está que la persona se sienta escuchada y, por otra, que la parte contraria también lo escuche de manera consciente. Cuando se presenta una situación muy ríspida, es necesario ser cuidadosos con el parafraseo, porque si una de las partes está muy enojada y empieza a decir alguna situación extrapolada, el mediador debe adaptar o suavizar la expresión durante el parafraseo, aunque sin llegar a modificar su esencia.

¿Qué impacto tienen los MASC en las personas que se acercan a estos procesos? El impacto es mucho, especialmente entre las personas que desconocen previamente la existencia y beneficios de los MASC. Cuando se les explica en qué consiste el procedimiento, los pasos a seguir y el reducido costo que implica, logra crearse un sentido de aprobación y confianza hacia estas alternativas. Les sorprende, por ejemplo, cuando se les dice que se gira una "invitación”, a diferencia de un citatorio; cuando se les explica que el mediador es un tercero imparcial que va a estar para escuchar a las dos partes y para facilitar el diálogo entre ellas, hasta lograr que ellos mismos tomen la resolución más ad hoc a su realidad. Así mismo, les genera certidumbre conocer que el procedimiento es confidencial.

Todo esto es trascendente para el desarrollo del proceso, pues el conocimiento y la confianza que les genera el procedimiento, ayudan a que la mediación sea de mayor calidad. Durante el proceso se realizan unas sesiones llamadas caucus, que son sesiones privadas, y la cercanía previa con el procedimiento resulta muy importante porque, en ese contexto, los participantes se sienten escuchados, valorados y reconocidos. En suma, el impacto en quienes recurren a esos métodos es positivo, porque conocen y valoran los beneficios de los MASC, además de lograr disipar sus conflictos de una manera mucho más expedita y pacífica que por las vías tradicionales.

¿Cuál ha sido el impacto que ha tenido el Centro, cuantas mediaciones, acuerdos o soluciones de conflictos se han logrado en este tiempo? En este periodo hemos llevado a cabo 80 mediaciones. De ese total, resultaron 16 convenios que trascendieron favorablemente ante los juzgados. ¿Porqué ante los juzgados? Porque en los divorcios por mutuo consentimiento, cuando hay hijos menores de edad, se da vista a la Procuraduría, pero eso no quiere decir que no se aplique la mediación, pues esta se realiza y luego llevamos el convenio ante el juzgado familiar. Así mismo, logramos validar 33 convenios ante el Instituto de Justicia Alternativa de Jalisco. 
En realidad, estos números representan un gran logro para la institución, especialmente al tratarse de un Centro de creación reciente y al desenvolverse principalmente en el ámbito universitario. Sin duda, una de las metas inmediatas para el Centro es dar mayor difusión a nuestra labor para lograr atraer más asesorías y encaminar las que correspondan hacia un proceso de mediación hasta lograr mayor número de convenios. Por otro lado, hay que destacar que sí ha logrado impactar a la comunidad del CUCEA, pues cada vez es más reconocida nuestra tarea y hay un mayor número de contactos por parte de los alumnos.

Respecto al impacto externo, también se puede hablar en términos positivos. Nuestra participación en los diversos medios de comunicación de la universidad, nos ha ayudado a ser más visibles fuera del campus y a través de ellos también hemos podido brindar numerosas asesorías entre un público muy amplio. Así mismo, nuestra colaboración con el Ayuntamiento de Zapopan, dentro del Centro Cultural Constitución, nos ha permitido llevar asesorías directas a aquellas colonias que tienen mayor demanda de soluciones de controversias. Todos estos resultados también nos han sido reconocidos por el IJA.

¿Dónde se ubica el Centro de Mediación número 156? El Centro es parte del Departamento de Ciencias Sociales y Jurídicas, que está asentado en el Centro Universitario de Ciencias Económico Administrativas de la Universidad de Guadalajara. En consecuencia, nuestra sede está dentro de dicho campus, exactamente en la planta baja del edificio " $M$ ”, en el espacio que ocuparía el aula número 108. Todo esto, localizado en el municipio de Zapopan, Jalisco, es decir, en el área metropolitana de Guadalajara.

Finalmente, puede agregarse que dentro de los MASC, independientemente de fomentar la cultura de paz, debemos impulsar la empatía en nuestra sociedad, porque al ser empáticos ayudamos al otro. En el Centro nos da muchísimo gusto que se acerquen los alumnos y digan "maestra, tengo un problema”, "maestra, mi mamá...", “maestra, mi prima tiene un problema”. Eso nos alegra porque saben que nosotros estamos para apoyarlos, entonces podemos ver que nuestras redes de soporte van creciendo.

Es muy importante para nosotros reforzar y ampliar nuestros proyectos de difusión. Una de las vías es seguir colaborando con la FIL — Feria Internacional del Libro de Guadalajara- y otra actividad que tenemos en proceso es hacer una caminata en pro de la cultura de la paz del CUCEA, por ejemplo, a través de la Vía Recreactiva Metropolitana, además de seguir promoviendo los servicios de nuestro Centro por múltiples medios y distintas formas. La idea es que más personas se enteren que hay otra manera de solucionar los conflictos. No dejamos todos los días de luchar, de soñar y de seguir implementando esta cultura de paz y la implementación de los MASC. Hay mucho por hacer, esto es sólo el principio y no bajaremos la guardia, especialmente en estos momentos tan difíciles que enfrenta el mundo y en los cuales es muy necesaria la conciliación. 